\title{
Brainstem tuberculous abscesses successfully treated by microsurgical excision: A case report and review of the literature
}

\author{
BAOFENG XU ${ }^{1}$, YANDONG ZHANG ${ }^{2}$ and JINLU YU ${ }^{1}$
}

Departments of ${ }^{1}$ Neurosurgery and ${ }^{2}$ Rheumatology, The First Hospital of Jilin University, Changchun, Jilin 130021, P.R. China

Received July 20, 2014; Accepted February 14, 2017

DOI: $10.3892 / 01.2017 .5782$

\begin{abstract}
In patients without systemic manifestations of tuberculosis, isolated brainstem tuberculous abscesses are infrequently observed. The present study reports a rare case of two cystic tuberculous abscesses involving the dorsal aspect of the medulla oblongata in an otherwise healthy 14-year-old Chinese girl, whose primary complaint was respiratory distress. These lesions were successfully treated using a microsurgical excision approach and anti-tuberculosis therapy. Although rare, tuberculous abscesses should be considered in the differential diagnosis of isolated cystic brainstem lesions. The results of the present study indicate that microsurgery, when combined with anti-tuberculosis therapy, is an effective and safe method to treat brainstem tuberculous abscesses.
\end{abstract}

\section{Introduction}

Tuberculosis is highly prevalent in developing countries, including China, particularly in the countryside (1). Involvement of the CNS frequently may be observed during tuberculosis infection (2). Tuberculous abscesses, however, are not frequently observed in patients without systemic manifestations of tuberculosis (3-5). Tuberculous brain abscesses are more frequently observed in patients positive for the human immunodeficiency virus (6). Early diagnosis of a tuberculous abscess remains difficult due to the complexities involved in differentiating these abscesses from other space-occupying lesions using a clinico-radiological profile (7). Although certain studies have recommended administering anti-tuberculosis therapy as a means of diagnosis (8-11), treatment is usually initiated only following histological confirmation of tuberculosis to avoid unnecessary treatment in patients with non-tubercular lesions. The present study reports a case of an otherwise healthy 14-year-old Chinese girl, who presented with a primary complaint of respiratory distress that was due to

Correspondence to: Dr Jinlu Yu, Department of Neurosurgery, The First Hospital of Jilin University, 71 Xinmin Avenue, Changchun Jilin 130021, P.R. China

E-mail: jinluyu@hotmail.com

Key words: tuberculous abscess, brainstem, microsurgical excision two cystic tuberculous abscesses. These lesions were successfully treated by microsurgical excision and anti-tuberculosis therapy.

\section{Case report}

A 14-year-old Chinese girl was admitted to the First Hospital of Jilin University (Changchun, China) on September 10, 2013 after complaining of intermittent nausea and headaches for one week and severe respiratory distress for two days. Physical examination of the patient revealed a respiratory rate of 30 breaths/min (normal range, $18-20$ breaths/min), a heart rate of 120 beats/min (normal range, $76-90$ beats/min), and a blood oxygen saturation of $100 \%$. The patient presented without a cough or fever. Neurological and laboratory examinations of the patient were normal. Serology for Borrelia burgdorferi, brucella, syphilis, toxoplasma gondii, cysticercosis and HIV was negative. A tuberculin skin test was not performed. No abnormalities were observed following a chest X-ray.

Unenhanced magnetic resonance imaging (MRI) revealed a poorly demarcated, oval shaped mass in the brainstem (Fig. 1A and B). An MRI fluid-attenuated inversion recovery scan showed mild dilatation of the lateral ventricles and edema in the surrounding area (Fig. 1C). Contrast-enhanced MRI revealed two, joined cystic masses in the brainstem that had well-defined hyperintense margins and hypointense centers and expansion to the fourth ventricle (Fig. 1D-F). The cystic lesions measured $2.2 \times 1.8 \times 1.6 \mathrm{~cm}$. A potential diagnosis of cystic space-occupying lesions in the dorsal aspect of the medulla oblongata was considered, pending surgical confirmation.

To obtain a histological diagnosis, and resolve potential neural tissue compression and cerebrospinal fluid pathway obstruction, a midline suboccipital craniectomy was subsequently performed. This procedure revealed a whitish, cystic and vascularized soft tissue mass with clearly defined margins in the dorsal aspect of the medulla oblongata. The walls of the cystic lesions were punctured and $\sim 2 \mathrm{ml}$ of viscous, yellowish fluid was aspirated. The aspirated fluid was negative for bacterial pathogens following a routine bacterial culture. Following aspiration of the fluid, the cystic lesions collapsed immediately, allowing safe removal of the cyst wall under magnification. During this operation, it was determined that the lesion had an intramedullary origin. The tissue was fixed with formalin for 12-48 h, and following gross examination 


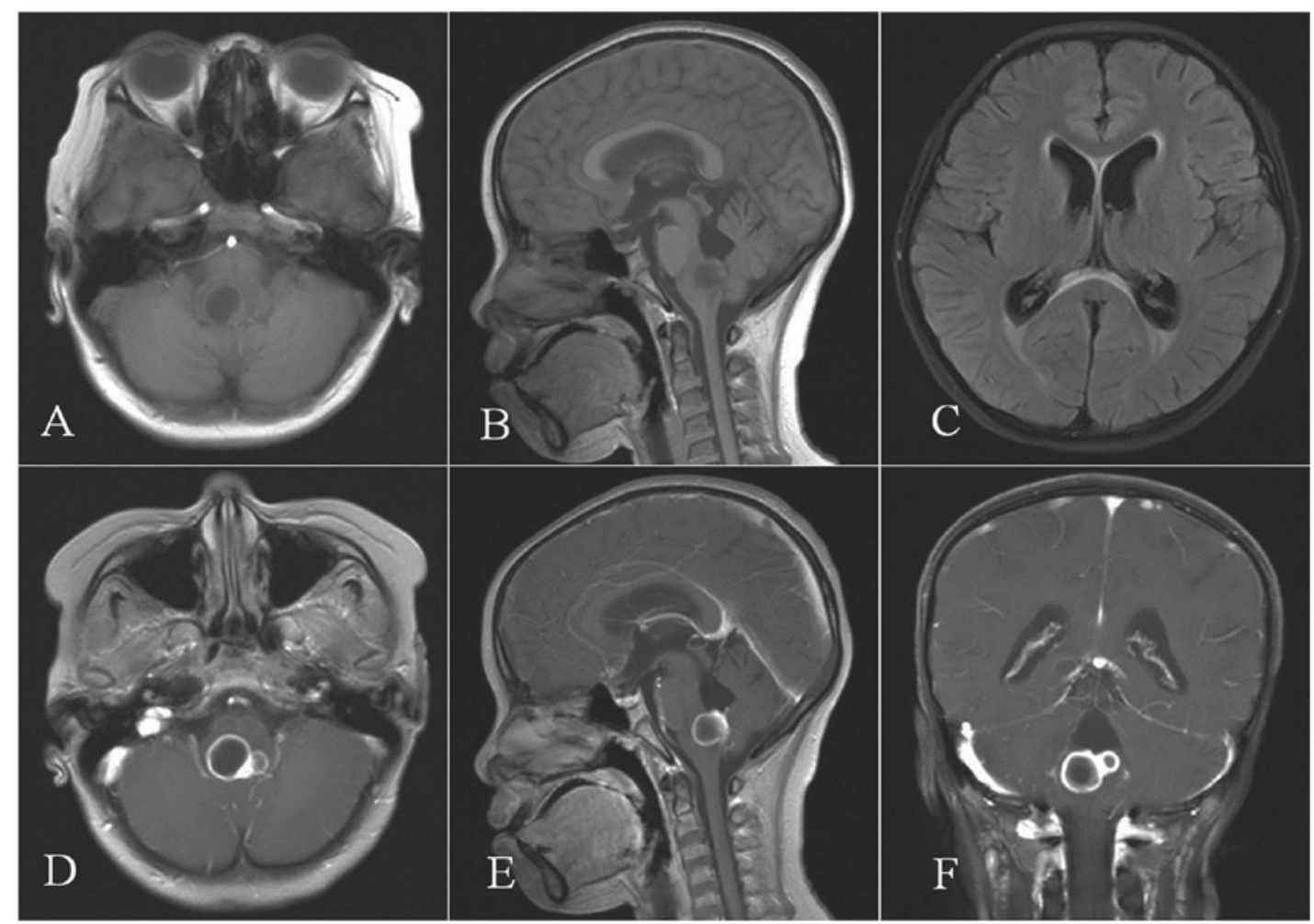

Figure 1. MRI scans. (A) Axial and (B) sagittal MRI revealed a poorly demarcated, oval shaped mass in the brainstem with mixed intensity. (C) Axial MRI fluid-attenuated inversion recovery scan revealed dilatation of the ventricular system and edema around the left anterior and occipital horns of the lateral ventricle. (D) Contrast-enhanced axial, (E) sagittal and (F) coronal MRI revealed a single large, well-defined, ring-enhancing cystic mass in the brainstem with a hypointense center joined by a smaller cyst, also possessing a hyperintense ring and hypointense center $(2.2 \times 1.8 \times 1.6 \mathrm{~cm})$. MRI, magnetic resonance imaging.

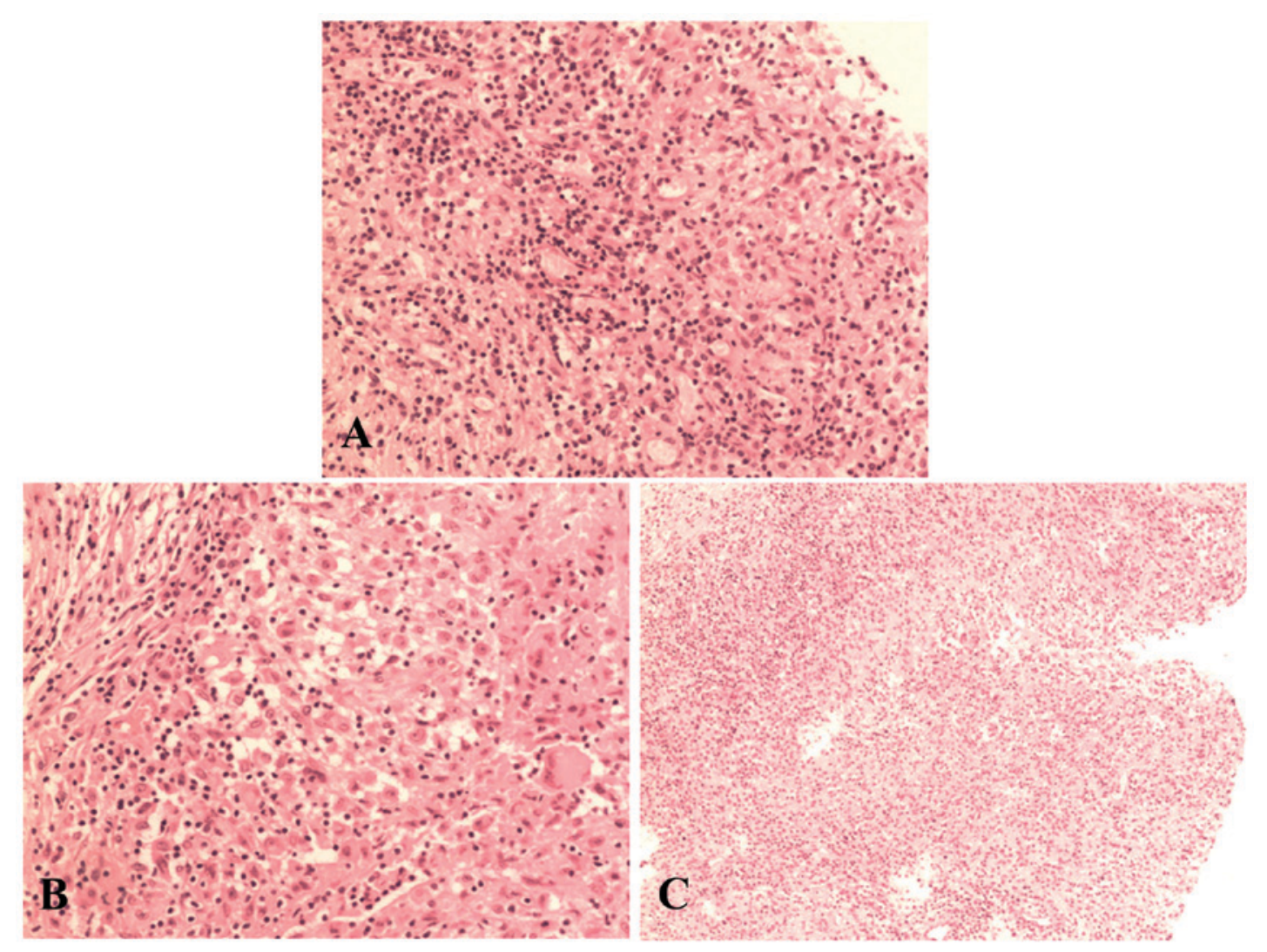

Figure 2. Histological examination using hematoxylin and eosin staining on the resected cyst wall showed (A) granulation tissue proliferation, epithelioid cell proliferation, acute and chronic inflammatory cell infiltration and Langhans giant cells in the cyst wall (magnification, $\mathrm{x} 400$ ). (B) Epithelioid cell proliferation and Langhans giant cells indicated tuberculous inflammation was present (hematoxylin and eosin staining; magnification, x400). (C) Acute and chronic inflammatory cells and foam cells indicated the presence of an abscess (hematoxylin and eosin staining; magnification, x200). 


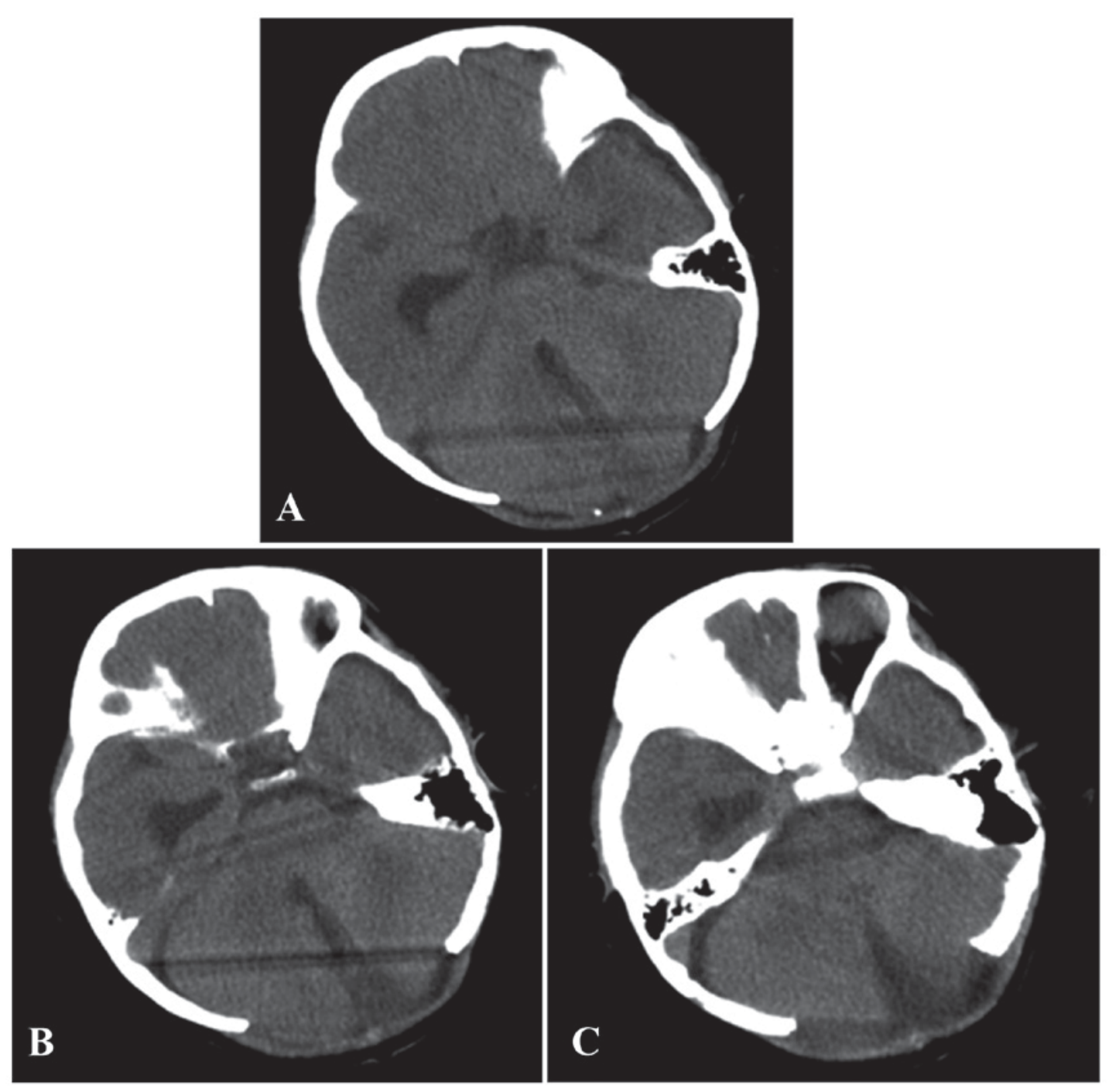

Figure 3. Computed tomography images. (A-C) A follow-up axial computed tomography following post-midline suboccipital craniectomy and microsurgical excision of the cystic tuberculomas revealed no recurrence of the abscesses.

the tissue was embedded in paraffin and cut (3-5 $\mu \mathrm{m})$ using the cryostat (Leica Biosystems Nussloch GmbH, Wetzlar, Germany). Hematoxylin and eosin staining was used to visualize the tissue architecture and a microscope (CX22; Olympus Corporation, Tokyo, Japan) was used to capture representative images. Histopathology of the resected tissues revealed epithelioid cell proliferation and Langhans giant cells, features that are consistent with tuberculous abscesses. Furthermore, these findings are inconsistent with features of cystic gliomas or pyogenic abscess (Fig. 2A-C). Thus, the present patient was diagnosed with tuberculous abscesses.

Postoperative recovery for the patient was uneventful and the respiratory distress and nausea gradually resolved. The patient exhibited no postoperative neurological sequelae. Following the surgery, the patient was given an anti-tuberculosis therapy of oral isoniazid ( $0.4 \mathrm{~g} / \mathrm{day})$, rifampicin (0.34 g/day) and pyrazinamide $(0.375 \mathrm{~g} /$ day; all purchased from Shanghai SINE Pharmaceutical Co., Ltd., Shanghai, China). Following a full recovery, the patient was discharged. While the patient was hospitalized, she underwent examination for signs of extra-pulmonary tuberculosis, but no evidence was observed. At a six-month follow-up visit, a computerized tomography (CT) scan revealed no recurrence of the tuberculous abscesses and the patient was asymptomatic (Fig. 3A-C). As a precaution, the patient received anti-tuberculosis therapy for one year. A total of three years post-operation, the patient is well and remains closely monitored.

Informed consent for the publication of the present report was obtained from the patient's family, and approval was provided from the Ethics Committee of the First Hospital of Jilin University (Changchun, China).

\section{Discussion}

Brainstem tuberculous abscesses are exceptionally rare (12). True tuberculous abscesses are defined as encapsulations of pus that contain acid-fast bacilli (AFB) without evidence of a mature tubercular granuloma (13). The following criteria were established by Whitener (14) to define a true tuberculous abscess: i) Macroscopic evidence of abscess formation within the brain parenchyma; ii) histological confirmation of acute and chronic inflammatory cells in the vascular granulation tissue within the abscess wall; and iii) bacteriological evidence of tuberculous origin. A cystic tuberculoma is another type of 
brainstem lesion that is similar in appearance to tuberculous abscesses. Tuberculous abscesses are distinct from tuberculomas, however, due to the caseation necrosis and liquefaction observed in tuberculomas (15). Cystic tuberculomas have previously undergone cystic degeneration and caseous liquefaction, which results in a liquid-filled cystic cavity that contains the components of a mature tuberculoma, including inflammatory cells, rare giant cells and epithelioid cells (16). Viable AFB may also be present in the cyst fluid, though this is not a requirement (17). Tyson et al (18) previously proposed that all tuberculous, encapsulated acute inflammatory exudates within the brain should be considered as tuberculous abscesses. The current consensus in the field, however, maintains that tuberculous abscesses and cystic tuberculomas are distinct entities (17).

In the present case, an MRI revealed that the tuberculous lesions were located in the medulla oblongata. A midline suboccipital craniectomy was performed and a whitish, cystic, vascularized soft-tissue mass with clearly defined margins was observed in the dorsal aspect of the medulla oblongata. Following puncturing of the walls of the cystic lesions, $\sim 2 \mathrm{ml}$ of viscous, yellowish fluid was aspirated from the abscesses. Histopathology of the resected tissues revealed proliferation of the granulation tissue, epithelioid cell proliferation, acute and chronic inflammatory cell infiltration, and Langhans giant cells in the cyst wall. Epithelioid cell proliferation and Langhans giant cells were indicative of tuberculous inflammation. Acute and chronic inflammatory cell infiltration, as well as the presence of foam cells, indicated that an abscess had formed. Thus, the patient was diagnosed with tuberculous abscesses. Furthermore, as tuberculomas form slowly and space in the brainstem is limited, clinical symptoms appear gradually over time, prior to liquefaction of the tuberculoma (19). In the present case, however, the patient had a rapid onset of symptoms, which were similar to those of a tuberculous abscess. The patient lives in China where tuberculosis is highly prevalent and involvement of the CNS is frequently observed (2).

Preoperative diagnosis of tuberculous abscess remains difficult. Using conventional imaging techniques, including MRI or CT scan, it is often impossible to conclusively differentiate tuberculous abscesses from cystic gliomas, pyogenic abscesses or other space-occupying lesions (20). The clinical presentation of a brainstem abscess is highly variable, and may include symptoms ranging from cranial nerve palsy, hemiparesis, dysphagia, speech and language disorders to more nonspecific symptoms, including nausea, headache and vomiting (21). The radiological findings may reveal a round or oval focal ring-enhancing lesion in the brainstem with peri-lesional effusion. However, this feature is also a hallmark of other fungal, parasitic and bacterial space-occupying lesions (22). Differential concentrations of proteins and amino acids contained within the lesion may allow an MRI to differentiate tuberculous brain abscesses and pyogenic abscesses (23). Regardless, histological confirmation is necessary for a conclusive diagnosis of tuberculosis in an isolated lesion when there is no evidence of systemic manifestations of tuberculosis infection.

Surgical excision and long term anti-tuberculosis treatment is considered the best course of treatment for these uncommon lesions $(3,24,25)$. Another treatment option utilizes puncture, drainage and aspiration of the lesion through burr holes; however, this approach has been reported in the literature to have varying efficacy (6). For brainstem abscesses, a microsurgical approach is advantageous because the abscess may be drained at the highest point of fluctuation with minimal damage to the surrounding brainstem tissue (26). Furthermore, direct visualization allows complete removal of the purulent content (27). While repeated aspirations with anti-tuberculosis therapy may also be considered as an option to manage a thin-walled cyst in an otherwise healthy patient, complete excision eliminated the risk of fluid re-accumulation within the cyst. In the present case, the cystic lesions demonstrated well-defined margins, and it was deemed safe to completely remove the cystic walls. Jiang et al (3) reported similarly favorable results following application of microsurgical excision and anti-tuberculosis therapy in a case of a tuberculous brainstem abscess. The morbidity of a brain abscess is unaffected by the surgical approach, and neurological sequelae are reported in up to $50 \%$ of patients, depending on the region of the brain affected (28). Early diagnosis and prompt treatment in the present patient resulted in a positive outcome.

The current case represents a highly unusual presentation of tuberculous abscesses. Although rare, tuberculous abscesses should be considered in the differential diagnosis of isolated cystic brainstem lesions, particularly in geographical regions where tuberculosis is prevalent. Histopathogical confirmation of tuberculosis is necessary to ensure administration of anti-tuberculosis therapy. Surgical excision and continued, long term anti-tuberculosis treatment is the preferred treatment for these lesions.

Tuberculous abscesses should be considered in the differential diagnosis of isolated cystic brainstem lesions. The results of the present study indicate that microsurgery combined with long-term anti-tuberculosis therapy is a safe and effective method to treat tuberculous abscesses of the brainstem.

\section{References}

1. Wang WB, Zhang H, Petzold M, Zhao Q, Xu B and Zhao GM: Cost-effectiveness of the Health X Project for tuberculosis control in China. Int J Tuberc Lung Dis 18: 939-945, 2014.

2. Li H, Liu W and You C: Central nervous system tuberculoma. J Clin Neurosci 19: 691-695, 2012.

3. Jiang YG, Chen J and Peng Y: Tuberculous brainstem abscess: Case report. Neurosurgery 65: E1206-E1207, 2009.

4. Prakash B, Mehta G, Gondal R, Kumar S and Malhotra V: Tuberculous abscess of the brain stem. Surg Neurol 32: 445-448, 1989.

5. Rajshekhar V and Chandy MJ: Tuberculomas presenting as isolated intrinsic brain stem masses. Br J Neurosurg 11: 127-133, 1997.

6. Vidal JE, Penalva de Oliveira AC, Bonasser Filho F, Schiavon Nogueira R, Dauar RF, Leite AG, Lins DL and Coelho JF: Tuberculous brain abscess in AIDS patients: Report of three cases and literature review. Int J Infect Dis 9: 201-207, 2005.

7. Cárdenas G, Soto-Hernández JL, Orozco RV, Silva EG, Revuelta R and Amador JL: Tuberculous brain abscesses in immunocompetent patients: Management and outcome. Neurosurgery 67, 1081-1087, 2010.

8. Talamás O, Del Brutto $\mathrm{OH}$ and García-Ramos G: Brain-stem tuberculoma. An analysis of 11 patients. Arch Neurol 46: 529-535, 1989.

9. Schoeman JF, Fieggen G, Seller N, Mendelson M and Hartzenberg B: Intractable intracranial tuberculous infection responsive to thalidomide: report of four cases. J Child Neurol 21: 301-308, 2006.

10. Muzumdar D, Balasubramaniam S and Mellkundi S: Tuberculous temporal brain abscess mimicking otogenic pyogenic abscess. Pediatr Neurosurgery 45: 220-224, 2009. 
11. Tekin R, Kaçar E, Cevik FC, Cinar K and Cevik R: Intracranial and intramedullary tuberculoma with intravertebral abscess manifestation under anti-tuberculous treatment: A case report. Clin Neurol Neurosurg 115: 1858-1860, 2013.

12. Roopesh Kumar VR, Gundamaneni SK, Biswas R and Madhugiri VS: Tuberculous cerebellar abscess in immunocompetent individuals. BMJ Case Rep 2012: bcr2012006984, 2012.

13. Kumar R, Pandey CK, Bose N and Sahay S: Tuberculous brain abscess: Clinical presentation, pathophysiology and treatment (In Children). Childs Nerv Syst 18: 118-123, 2002.

14. Whitener DR: Tuberculous brain abscess. Report of a case and review of the literature. Arch Neurol 35: 148-155, 1978.

15. Dastur HM: A tuberculoma review with some personal experiences. I Brain. Neurol India 20: 111-126, 1972.

16. Sinh G, Pandya SK and Dastur DK: Pathogenesis of unusual intracranial tuberculomas and tuberculous space-occupying lesions. J Neurosurg 29: 149-159, 1968.

17. Sridhar K, Ramamurthi B and Vasudevan MC: Cystic tuberculomas of the brain - two case reports. Neurol Med Chir (Tokyo) 44: 438-441, 2004.

18. Tyson G, Newman P and Strachan WE: Tuberculous brain abscess. Surg Neurol 10: 323-325, 1978.

19. Sarkar S, Patra C and Dasgupta MK: Bilateral ptosis without upward gaze palsy: Unusual presentation of midbrain tuberculoma. J Neurosci Rural Pract 8: 129-132, 2017.

20. Lyons JL, Neagu MR, Norton IH and Klein JP: Diffusion tensor imaging in brainstem tuberculoma. J Clin Neurosci 20: $1598-1599,2013$.
21. Fuentes S, Bouillot P, Regis J,Lena G and Choux M: Management of brain stem abscess. Bf J Neurosurg 15: 57-62, 2001.

22. Kagawa R, Okada Y, Shima T, Nishida M and Yamane K Neuroimaging findings of the development and resolution of solitary brainstem abscess: Characteristics of neuroimagings in the early stage of brainstem abscess and importance of surgical management for brainstem abscess - case report. Neurol Med Chir (Tokyo) 39: 621-624, 1999.

23. Gupta RK, Vatsal DK, Husain N, Chawla S, Prasad KN, Roy R, Kumar R, Jha D and Husain M: Differentiation of tuberculous from pyogenic brain abscesses with in vivo proton MR spectroscopy and magnetization transfer MR imaging. AJNR Am J Neuroradiol 22: 1503-1509, 2001.

24. García-Moncó JC, Gómez Beldarrain M, Fernández Cantón G, Capelastegui A and Collazos J: Resolution of a brainstem abscess through antituberculous therapy. Neurology 49: 265-267, 1997.

25. Hernández JL, López PC and Escobar A: Resolution of a brainstem abscess through antituberculosis therapy. Neurology 50: 1929-1930, 1998.

26. Tayebi Meybodi A, Lawton MT, Tabani H and Benet A: Tonsillobiventral fissure approach to the lateral recess of the fourth ventricle. J Neurosurg: Oct 28, 2016 (Epub ahead of print).

27. Suzer T, Coskun E, Cirak B, Yagci B and Tahta K: Brain stem abscesses in childhood. Childs Nerv Syst 21: 27-31, 2005.

28. Hall WA: Cerebral infectious processes. In: Neurosurgical emergencies. Loftus CM (ed). Vol 1. 1st edition. American Association of Neurological Surgeons Publications, Park Ridge, IL: pp165-182, 1994. 